\title{
Ranges of protons in biological targets
}

\author{
Márius Pavlovič, ${ }^{*}$ Andreas Hammerle ${ }^{* *}$
}

\begin{abstract}
The paper introduces a simple fitting function for quick assessment of proton ranges in biological targets and human tissues. The function has been found by fitting an extensive data set of Monte Carlo proton ranges obtained with the aid of the SRIM-2013 code. The data has been collected for 28 different targets at 8 energies in the interval from $60 \mathrm{MeV}$ to $220 \mathrm{MeV}$. The paper shows that at a given kinetic proton-beam energy, the Monte Carlo ranges can be satisfactorily fitted by a power function that depends solely on the target density. This is a great advantage for targets, for which the exact chemical composition is not known, or the mean ionizing potential is not reliably known. The satisfactory fit is meant as the fit that stays within the natural range straggling of the Monte Carlo ranges. In the second step, the energy-scaling yielding a universal fitting formula for proton ranges as a function of proton-beam energy and target density is introduced and discussed.
\end{abstract}

This paper is an extended version of the contribution presented at the APCOM2017 conference.

K e y w o r d s: biological targets, proton range, proton therapy, range straggling, SRIM

\section{Introduction}

Ion therapy is a promising cancer treatment modality for specific types of tumours. Its application requires technological development in the field of accelerator technology [1-3], beam transport [4-7], radiobiology, dosimetry, treatment planning, etc. Many of these development fields are based on the physics of ion interaction with matter, which is tightly connected to the precise knowledge of ion ranges in different biological targets. The present paper deals with systematic calculation of therapeutic protonbeam ranges in different human tissues. The ranges are calculated by the Monte Carlo module of the SRIM-2013 code (SRIM=Stopping and Ranges of Ions in Matter) [8]. The human tissues that are selected from the SRIM-2013 built-in compound dictionary are characterized by their density. The tissue density is used as the only variable in the range-fitting formula at a given proton-beam energy. The range-to-density data are collected for eight protonbeam energies covering the clinically relevant energy interval from $60 \mathrm{MeV}$ to $220 \mathrm{MeV}$. This made it possible to find also energy-scaling for the fitting parameters belonging to individual energy sets. The fitting functions have been tested by comparing the fitted ranges with the Monte Carlo ranges. The paper shows that the therapeutic proton-beam ranges in biological and human tissues can be satisfactorily assessed by an extremely simple function of the tissue density. The result can be used for quick assessment of the proton-beam range in human tissues at many clinical and research applications like phantom design and phantom experiments, design and preparation of quality-assurance set-ups, design and prepara- tion of in-vitro biological experiments with human tissues irradiated by proton beams, in-vivo animal experiments, etc.

\section{Materials and methods}

The well-established and widely recognized computer program SRIM-2013 (SRIM=Stopping and Ranges of Ions in Matter) [8] was used to calculate proton ranges in the energy interval from $60 \mathrm{MeV}$ to $220 \mathrm{MeV}$ [9] in 28 different biological targets taken from the built-in SRIM compound dictionary. The targets were selected from the category called "Biological materials - Human". The SRIM compound dictionary provides information about chemical composition of each material and its density. We were looking for the range-to-density dependence without taking into account the target chemical composition, because the exact chemical composition of individual tissues and organ structures varies in time and can never be precisely known in clinical practice at the particular moment of patient irradiation. The density is a material parameter that is more stable and can be better correlated with the so-called CT numbers (Hounsfield units) gained from the CT images [10]. Hence the main question of interest is: "Is it possible to predict the ion range as a function of the target density in a material with "unknown" chemical composition?" It must, however, be pointed out that this "unknown" chemical composition is meant within a group of materials with similar chemical composition, like different human tissues. Table 1 summarizes the results of all simulations.

* Faculty of Electrical Engineering and Information Technology, Slovak University of Technology in Bratislava, Ilkovičova 3, 81219 Bratislava, Slovakia, marius.pavlovic@stuba.sk ** University of Applied Sciences in Wiener Neustadt, Johannes-Gutenberg-Strasse 3, A-2700 Wiener Neustadt, Austria 
Table 1. Proton-beam ranges in different human tissues taken from the SRIM built-in compound dictionary

\begin{tabular}{|c|c|c|c|c|c|c|c|c|c|}
\hline \multirow{5}{*}{ Tissue } & \multirow{5}{*}{$\begin{array}{l}\text { Density } \\
\left(\mathrm{g} / \mathrm{cm}^{3}\right)\end{array}$} & \multicolumn{8}{|c|}{ Proton-beam kinetic energy $(\mathrm{MeV})$} \\
\hline & & 60 & 100 & 120 & 140 & 150 & 180 & 200 & 220 \\
\hline & & \multicolumn{8}{|c|}{ Average natural range straggling ( $\%$ of the range) } \\
\hline & & 1.43 & 1.38 & 1.36 & 1.36 & 1.36 & 1.35 & 1.33 & 1.32 \\
\hline & & \multicolumn{8}{|c|}{ SRIM Monte Carlo range $(\mathrm{mm})$} \\
\hline Water (liquid) & 1 & 30.4 & 76.1 & 105 & 138 & 155 & 213 & 256 & 301 \\
\hline Human blood, ICRU & 1.06 & 29 & 72.5 & 100 & 131 & 148 & 203 & 244 & 287 \\
\hline Human skin & 1.09 & 28.2 & 70.4 & 97.3 & 127 & 144 & 198 & 237 & 279 \\
\hline Skeletal muscle & 1.05 & 29.3 & 73.2 & 101 & 132 & 149 & 205 & 246 & 290 \\
\hline Skeleton-cartilage & 1.1 & 28.3 & 70.7 & 97.7 & 128 & 144 & 198 & 238 & 280 \\
\hline Skeleton-spongiosa & 1.18 & 26.7 & 66.7 & 92.2 & 120 & 136 & 187 & 224 & 264 \\
\hline Skeleton-red marrow & 1.03 & 29.5 & 73.8 & 102 & 133 & 151 & 207 & 248 & 292 \\
\hline Skeleton-yellow marrow & 0.98 & 30.4 & 76.2 & 105 & 138 & 156 & 214 & 257 & 302 \\
\hline Cortical bone, adult & 1.92 & 18.1 & 45.2 & 62.4 & 81.8 & 92.2 & 126 & 151 & 178 \\
\hline Cortical bone, age $6-13$ & 1.83 & 18.9 & 47 & 64.8 & 85 & 95.9 & 131 & 157 & 185 \\
\hline Cortical bone, age $2-5$ & 1.8 & 19.1 & 47.7 & 65.8 & 86.2 & 97.3 & 133 & 160 & 188 \\
\hline Perinatal rhesus monk & 1.4 & 23.3 & 58.2 & 80.4 & 105 & 119 & 163 & 195 & 230 \\
\hline Mammary gland, \#1 & 0.99 & 30.5 & 76.2 & 105 & 138 & 156 & 214 & 257 & 302 \\
\hline Mammary gland, \#2 & 1.02 & 29.8 & 74.5 & 103 & 135 & 152 & 209 & 251 & 296 \\
\hline Mammary gland, \#3 & 1.06 & 29 & 72.4 & 100 & 131 & 148 & 203 & 244 & 287 \\
\hline Spleen & 1.09 & 28.2 & 70.5 & 97.4 & 127 & 144 & 198 & 237 & 279 \\
\hline Testis & 1.04 & 29.4 & 73.6 & 101 & 133 & 150 & 206 & 248 & 291 \\
\hline Thyroid & 1.05 & 29.2 & 73 & 100 & 132 & 149 & 205 & 246 & 289 \\
\hline Trachea & 1.06 & 29 & 72.6 & 100 & 131 & 148 & 204 & 244 & 287 \\
\hline Ovary & 1.05 & 29.2 & 73 & 100 & 132 & 149 & 205 & 245 & 289 \\
\hline Pancreas & 1.04 & 29.1 & 72.7 & 100 & 131 & 148 & 204 & 245 & 288 \\
\hline Prostate & 1.04 & 29.5 & 73.7 & 101 & 133 & 150 & 207 & 248 & 292 \\
\hline Urinary bladder-urine & 1.02 & 29.9 & 74.9 & 103 & 135 & 153 & 210 & 252 & 296 \\
\hline Urinary bladder, empty & 1.04 & 29.5 & 73.7 & 101 & 133 & 150 & 207 & 248 & 292 \\
\hline Urinary bladder, full & 1.03 & 29.7 & 74.2 & 102 & 134 & 151 & 208 & 250 & 294 \\
\hline Adipose tissue & 0.92 & 32.2 & 80.7 & 111 & 146 & 165 & 227 & 272 & 320 \\
\hline Bone-cortical, ICRP & 1.85 & 18.5 & 46 & 63.5 & 83.2 & 93.9 & 128 & 154 & 181 \\
\hline Muscle-skeletal, ICRP & 1.04 & 29.6 & 74.1 & 102 & 134 & 151 & 208 & 249 & 293 \\
\hline
\end{tabular}

\section{Results}

\subsection{Fitting the individual energy sets}

Data in Tab. 1 represent the mean-value of the range distribution and the natural range straggling shows one standard deviation (square root of the variance) of the range distribution. These data are presented in graphical form in Fig. 1. Shown points are the ranges calculated by SRIM. Let us call them Monte Carlo ranges, $R_{\mathrm{MC}}$. The vertical error bars represent the range straggling defined as the square root of the variance of the range distribution. Let us call it natural range straggling. This parameter is automatically calculated by SRIM during the Monte Carlo simulation. That is why we will use notation $S_{\mathrm{MC}}$ for the natural range straggling. Each range is accompanied by its individual value of the natural range straggling. The natural range straggling can be expressed either directly in millimeters (as in Fig. 1) or in percentage of the Monte Carlo range, $S_{\mathrm{MC}} / R_{\mathrm{MC}} \times 100 \%$ (as in Tab. 1, where the natural range straggling is averaged over all data points belonging to the same energy). The relative natural range straggling is almost the same at all proton-beam energies ranging from $1.43 \%$ to $1.32 \%$ at $60 \mathrm{MeV}$ and $220 \mathrm{MeV}$, respectively. The data sets corresponding to different energies $(60 \mathrm{MeV}, 100 \mathrm{MeV}, 120$ $\mathrm{MeV}, 140 \mathrm{MeV}, 150 \mathrm{MeV}, 180 \mathrm{MeV}, 200 \mathrm{MeV}$, and 220 $\mathrm{MeV}$ ) can be clearly recognized.

The solid lines represent the best analytical fit of the Monte Carlo ranges at given energy. We found out that the best fit was achieved by a power function in the form

$$
R_{\mathrm{FIT}}=\frac{A}{\rho^{B}},
$$

where $R_{\text {FIT }}$ is the fitted range in mm, $\rho$ is the tissue density in $\mathrm{g} / \mathrm{cm}^{3}, A$ is a coefficient and $B$ is an exponent. 


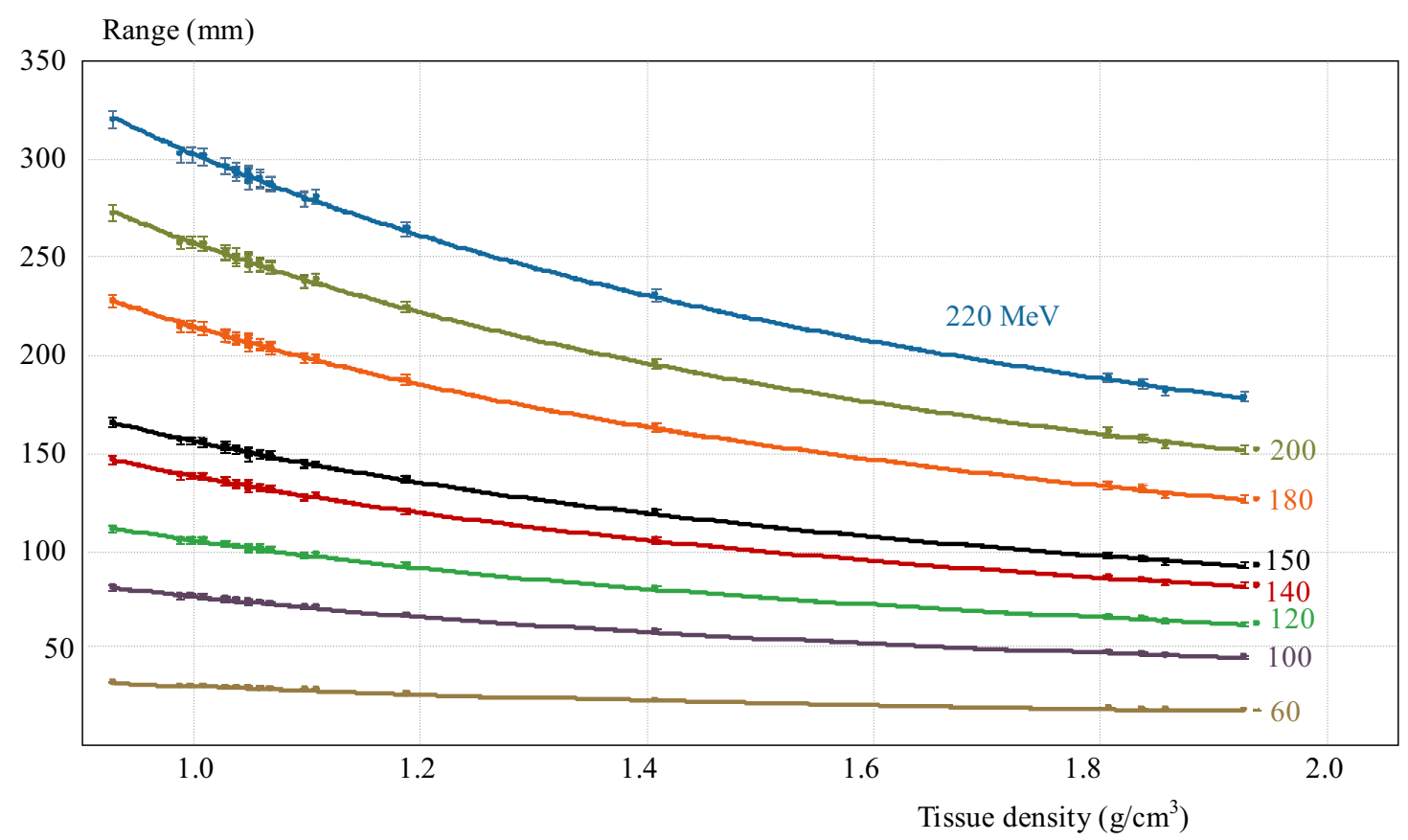

Fig. 1. Proton - beam ranges as a function of target density calculated by SRIM for different proton - beam energies from $60 \mathrm{MeV}$ to $220 \mathrm{MeV}$

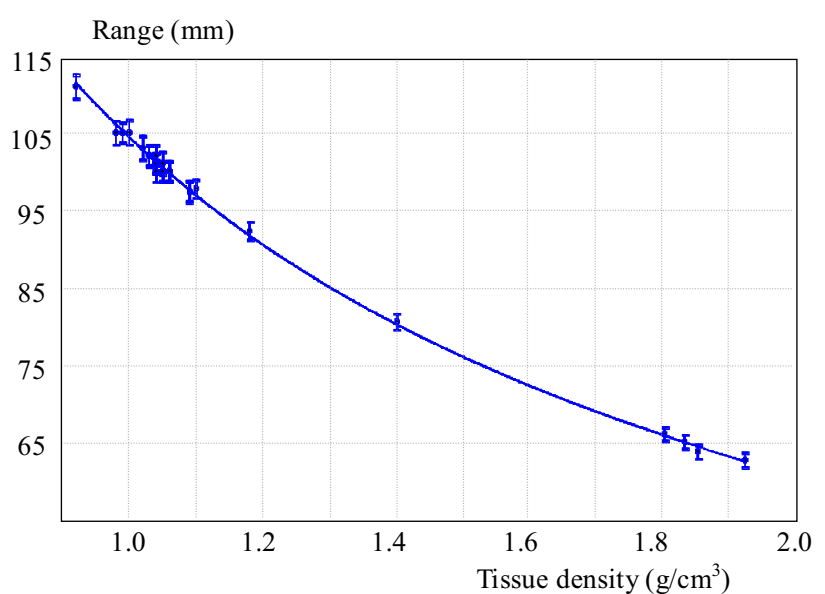

Fig. 2. Proton - beam ranges as a function of target density calculated by SRIM for the proton - beam energy of $120 \mathrm{MeV}$

The values of the $A$ and $B$ fit-parameters are listed in Tab. 2 together with the statistical $R^{2}$-parameter characterizing the fit quality. These parameters were gained by the "Trendline" routine of MS-EXCEL. It can be seen from Tab. 2, that according to the $R^{2}$-criterion, the worst fit was achieved for the $120 \mathrm{MeV}$ data set. This data is shown in Figure 2. This data set represents the worst fit according to the $R^{2}$ - criterion. Nevertheless, the fitted ranges stay within the natural range straggling at all data points.

Although the $R^{2}$-parameter is commonly used to characterize the fit quality, we were looking for a physicsbased criterion and decided to test whether the fitting function stayed within the natural range straggling. Each Monte Carlo range is accompanied by its individual value of the natural range straggling, $S_{\mathrm{MC}}$ (the values quoted in

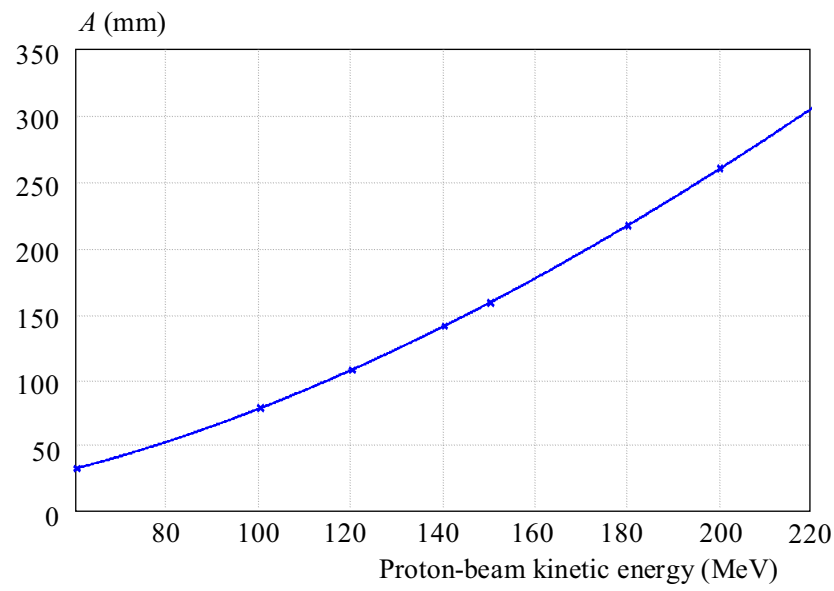

Fig. 3. The A (E) data and the 4 th - order polynomial fitting function according to (3)

Tab. 1 are averaged over all data points belonging to the same proton-beam energy). For each Monte Carlo range, we calculated the corresponding fitted value, $R_{\mathrm{FIT}}$, using (1) and values from Tab. 2. After that, the deviation of the fitted range from the Monte Carlo range, $D$, was calculated for each data point as

$$
D=\frac{\left|R_{\mathrm{MC}}-R_{\mathrm{FIT}}\right|}{R_{\mathrm{MC}}} 100 \%
$$

Finally, the deviation, $D$, was compared with the relative natural range straggling, $S_{\mathrm{MC}}$, as $S_{\mathrm{MC}}-D$. In case of the positive result, the fitting function stays inside the "error bar" representing the natural range straggling. In case of the negative result, the fitting function extends out of the natural range straggling. Results of the above described test are summarized in Tab. 3. 
Table 2. The fit-parameters of the power function fitting the Monte Carlo proton-beam ranges

\begin{tabular}{|c|c|c|c|c|}
\hline \multirow{2}{*}{ Parameter } & \multicolumn{4}{|c|}{ Proton-beam kinetic energy $(\mathrm{MeV})$} \\
\hline & 60 & 100 & 120 & 140 \\
\hline $\bar{A}$ & 30.31 & 75.81 & 104.39 & 136.96 \\
\hline$B$ & 0.788 & 0.795 & 0.79 & 0.794 \\
\hline$R^{2}$ & 0.999 & 0.999 & 0.998 & 0.999 \\
\hline \multirow{2}{*}{ Parameter } & \multicolumn{4}{|c|}{ Proton-beam kinetic energy $(\mathrm{MeV})$} \\
\hline & 150 & 180 & 200 & 220 \\
\hline$A$ & 154.7 & 212.82 & 255.27 & 300.34 \\
\hline$B$ & 0.795 & 0.806 & 0.805 & 0.804 \\
\hline$R^{2}$ & 0.999 & 0.999 & 0.999 & 0.999 \\
\hline
\end{tabular}

Table 3. Results of the fitting test in terms of fit-deviation versus the natural range straggling

\begin{tabular}{lccccc}
\hline & \multicolumn{5}{c}{ The worst data point } \\
Energy & \multicolumn{2}{c}{ Average } & \multicolumn{2}{c}{ Individual } \\
$(\mathrm{MeV})$ & $S_{\mathrm{MC}}$ & $D$ & $S_{\mathrm{MC}}$ & $D$ & $S_{\mathrm{MC}}-D$ \\
& $(\%)$ & $(\%)$ & $(\%)$ & $(\%)$ & $(\%)$ \\
\hline 60 & 1.43 & 0.38 & 1.54 & 1.3 & 0.24 \\
100 & 1.38 & 0.39 & 1.37 & 1.06 & 0.31 \\
120 & 1.36 & 0.41 & 1.45 & 1.21 & 0.24 \\
140 & 1.36 & 0.4 & 1.42 & 1.34 & 0.08 \\
150 & 1.36 & 0.31 & 1.47 & 1.32 & 0.15 \\
180 & 1.35 & 0.37 & 1.37 & 1.26 & 0.11 \\
200 & 1.33 & 0.37 & 1.40 & 1.00 & 0.40 \\
220 & 1.32 & 0.37 & 1.33 & 1.19 & 0.14 \\
\hline
\end{tabular}

no points were found out of $S_{\mathrm{MC}}$

It can be seen that the fitting function stays inside the straggling bars at all data points. The largest deviation of the fitted range from the Monte Carlo range is less than $1.4 \%$ (at $140 \mathrm{MeV}$ ) and the average deviation represents less than $28 \%$ of the average natural range straggling.

\subsection{Energy - scaling}

So far, the parameters of the analytical fitting function had to be found individually for each proton-beam energy set. However, it shall be possible to find also an energy-scaling based on some correlation between the fitting parameters and the proton-beam energy. The situation is rather straightforward for the coefficient $\mathrm{A}$ that has an obvious physical meaning. It is the fitted range in water (1), $R_{\mathrm{FIT}}=A(\mathrm{~mm})$ if $\rho=1 \mathrm{~g} / \mathrm{cm}^{3}$. The range in water as a function of the proton-beam energy is a well-studied dependence [11-14]. We adopted the model proposed in Ref. [14] and fitted the $A(E)$ data by the 4 th-order polynomial function

$$
A(E)=a E^{4}+b E^{3}+c E^{2}+d E,
$$

where $E$ is the proton-beam kinetic energy in $\mathrm{MeV}$ and the $a, b, c$, and $d$ coefficients have been gained again by the MS EXCEL "Trendline" routine: $a=6.75 \times 10^{-9}$, $b=-9.75 \times 10^{-6}, c=7.60 \times 10^{-3}$ and $d=8.39 \times 10^{-2}$. The $R^{2}$-parameter of this fit is 0.999976 . Figure 3 shows the $A(E)$ data points together with the above described fitting function given by (3). It can be seen that the fitting model adopted from [14] fits extremely well our data.

In contrast to the A-coefficient, the B-exponent does not show any reasonable dependence on the proton-beam energy (see Tab. 2). Nevertheless, all B-coefficient values are very close to each other. That is why we replaced all of them - purely intuitively - by the average value of 0.797 . After applying the energy-scaling, the fitting parameters from Tab. 2 changed to the fitting parameters according to Tab. 4 containing the fitting parameters calculated according to (3) and the average value of the B-exponent.

Table 4. The fit-parameters of the power function obtained by energy-scaling of the fit-parameters from Tab. 2

\begin{tabular}{lcccc}
\hline \multirow{2}{*}{ Parameter } & \multicolumn{4}{c}{ Proton-beam kinetic energy $(\mathrm{MeV})$} \\
A & 60 & 100 & 120 & 140 \\
B & 30.414 & 75.495 & 104.371 & 137.039 \\
Parameter & \multicolumn{4}{c}{ Proton-beam kinetic energy } \\
& 150 & 180 & 200 & 220 \\
A & 154.703 & 212.616 & 255.02 & 300.209 \\
B & \multicolumn{4}{c}{0.797} \\
\hline
\end{tabular}

We have repeated the fit-quality test in the same manner as described in the previous subsection using the fitting parameters from Tab. 4 instead of the fitting parameters from Tab. 2. Results of this test are shown in Tab. 5 .

Table 5. Results of the fitting test in terms of fit-deviation versus the natural range straggling using the fitting parameters from Tab. 4 resulting from the energy-scaling

\begin{tabular}{lcccccc}
\hline & \multicolumn{5}{c}{ The worst data point } \\
Energy & \multicolumn{4}{c}{ Average } & \multicolumn{2}{c}{ Individual } \\
$(\mathrm{MeV})$ & $N$ & $S_{\mathrm{MC}}$ & $D$ & $S_{\mathrm{MC}}$ & $D$ & $S_{\mathrm{MC}}-D$ \\
& & $(\%)$ & $(\%)$ & $(\%)$ & $(\%)$ & $(\%)$ \\
\hline 60 & 1 & 1.43 & 0.4 & 1.54 & 1.67 & -0.13 \\
100 & 0 & 1.38 & 0.59 & 1.3 & 1.25 & 0.05 \\
120 & 0 & 1.36 & 0.46 & 1.24 & 0.99 & 0.25 \\
140 & 0 & 1.36 & 0.4 & 1.42 & 1.39 & 0.03 \\
150 & 0 & 1.36 & 0.32 & 1.47 & 1.31 & 0.16 \\
180 & 1 & 1.35 & 0.37 & 1.37 & 1.73 & -0.36 \\
200 & 1 & 1.33 & 0.39 & 1.4 & 1.42 & -0.02 \\
220 & 1 & 1.32 & 0.37 & 1.33 & 1.58 & -0.25 \\
\hline \multicolumn{7}{l}{$N=$ number of poits found out of $S_{\mathrm{MC}}$}
\end{tabular}


In this case, the largest deviation of the fitted range from the Monte Carlo range is less than $1.8 \%$ (at 180 $\mathrm{MeV}$ ) and the average deviation represents less than 31 $\%$ of the average natural range straggling.

\section{Discussion and conclusions}

It has been shown that for a group of biological materials and human tissues, the proton-beam range can be satisfactorily assed by an analytical function with the only variable - the target density. The best form of this analytical function is a power function with two parameters: the coefficient and the exponent. These parameters were found for 8 energies in the clinically relevant energy interval from $60 \mathrm{MeV}$ to $220 \mathrm{MeV}$ corresponding to the proton-beam range in a water-equivalent tissue from 30.4 $\mathrm{mm}$ up to $301 \mathrm{~mm}$, respectively. The fitting was done with the aid of the built-in "Trendline" routine of MS-EXCEL. The ranges were calculated with the SRIM-2013 code and the biological materials were selected from its compound dictionary - Biological materials - Human.

The fit quality was evaluated by the statistical $R^{2}$ parameter that is listed in Tab. 2. The average value of the $R^{2}$-parameter is 0.99915 . The worst value is 0.99898 at the proton-beam energy of $120 \mathrm{MeV}$.

In addition to this, we tested the analytical fitting function with respect to the natural range straggling representing 1-sigma value of the range distribution. The fitting function stays inside the natural range straggling at all data points. The average range straggling for all data points is $1.36 \%$ of the range. The average deviation of the fitted range from the Monte Carlo range is $0.38 \%$ of the Monte Carlo range. This means that on average, the fitting function stays inside $28 \%$ of the natural range straggling, which is an excellent agreement. The worst data point is contained in the energy set belonging to $140 \mathrm{MeV}$. The natural range straggling of this particular point is $1.42 \%$ of the Monte Carlo range. The deviation of the fitted range from the Monte Carlo range is 1.34 $\%$ of the Monte Carlo range. The difference between the natural range straggling and the deviation is $0.08 \%$ of the Monte Carlo range.

In the next step, we found an energy-scaling that allows assessing the proton ranges in biological targets as a function of the kinetic proton-beam energy and the target density. The energy-scaling was based on the 4th-order polynomial fit of the power-function coefficients and average value of the power-function exponents. The energyscaling did worsen slightly the fit quality. The average deviation became $0.41 \%$ of the Monte Carlo range, which corresponds to $31 \%$ of the average natural range straggling. Although this is still a very good agreement, four fitted ranges got out of the natural range straggling. The worst point has been found at $180 \mathrm{MeV}$ with deviation of $1.73 \%$ of the Monte Carlo range, whereas the natural range straggling at this point is $1.37 \%$ of the Monte Carlo range.
As the final conclusion, the energy-scaled fitting formula assesses the ranges with accuracy below $1.8 \%$ of the Monte Carlo range (see Tab. 5, $180 \mathrm{MeV}$ ), whereas the fitting of the individual energy sets can be done with accuracy better than $1.4 \%$ of the Monte Carlo range (see Tab. 3, $140 \mathrm{MeV}$ ). The biological human targets have been our favorite group of materials selected for this study mainly due to several reasons: (1) the SRIM compound dictionary contains the largest set of materials in this particular category; (2) these materials have rather similar chemical composition and reasonably narrow densityspan; (3) the problem is very relevant from the practical point of view. In real life, getting the exact chemical composition of different organ structures in-vivo is not possible, but their density can be well correlated to the CT-numbers.

Nevertheless, there are other suitable candidates that may profit from our results. The first group of materials could be so-called metallic glasses, especially the softmagnetic metallic alloys. They also feature similar chemical composition $[15,16]$ and the ion-range data are needed for radiation hardness studies of these materials against ion irradiation [17-21]. The second possible candidates could be steels; a large family of materials made of the same iron bulk material with different additive elements and processed by different technological treatments. They are important construction materials in nuclear industry $[22,23]$ (particle accelerators, reactor vessels, etc.). That is why they are also subject to radiation hardness studies against ion and neutron irradiation requiring quick and reasonably accurate assessment of ion ranges [24-27].

\section{Acknowledgements}

This work was financially supported by the Scientific Grant Agency of the Ministry of Education, Science, Research and Sport of Slovak Republic No. VEGA 2/0152/16 and VEGA 1/0863/17.

\section{REFERENCES}

[1] H. Owen, R. MacKay, K. Peach and S. Smith, "Hadron accelerators for radiotherapy", Contemporary Physics, 2014, vol. 55, no. $2,55-74$.

[2] H. Owen, D. Holder, J. R. Alonso and R. MacKay, "Technologies for delivery of proton and ion beams for radiotherapy (Review)", International Journal of Modern Physics A, 2014, vol. 29, no.14, article number 1441002, 1-39.

[3] H. Owen, A. Lomax and S. Jolly, "Current and future accelerator technologies for charged particle therapy", Nuclear Instruments and Methods Physics Research A, 2016, vol. 809, 96-104.

[4] M. Pavlovič, "A design of a rotating gantry for non-symmetric ion-therapy beams", Nuclear Instruments and Methods Physics Research A, 1999, vol. 438, 548-559.

[5] M. Pavlovič, E. Griesmayer and R. Seemann, "Beam-transport study of an isocentric rotating ion gantry with minimum number of quadrupoles", Nuclear Instruments and Methods Physics Research A, 2005, vol. 545, 412-426.

[6] J. Kim and M. Yoon, "Design study of a superconducting gantry for carbon beam therapy", Journal of the Korean Physical Society, 2016, vol. 69, no. 6, 1048-1052. 
[7] J. Bokor and M. Pavlovič, "An ion-optical design study of a carbon-ion rotating gantry with a superconducting final bending magnet", Nuclear Instruments and Methods Physics Research A, 2016, vol. 812, 122-133.

[8] J. F. Ziegler, M. D. Ziegler and J. P. Biersack, "SRIM - The stopping and range of ions matter (2010)", Nuclear Instruments and Methods Physics Research B, 2010, vol. 268, no. 11-12, 1818-1823.

[9] E. Griesmayer, T. Schreiner and M. Pavlovič, "The MedAustron project", Nuclear Instruments and Methods Physics Research B, 2007, vol. 258, no. 1, 134-138.

[10] M. Pavlovič and A. Hammerle, Ion Ranges Different Human Tissues, Lambert Academic Publishing, Saarbrücken, Germany, 2017, ISBN: 978-3-330-01715-3.

[11] W. Ulmer and B. Schaffner, "Foundation of an analytical proton beamlet model for inclusion a general proton dose calculation system", Radiation Physics and Chemistry, 2011, vol. 80, 378-389.

[12] E. V. Bellinzona, M. Ciocca, A. Embriaco, A. Ferrari, A. Fontana, A. Mairani, K. Parodi, A. Rotondi, P. Sala and T. Tessonnier, "A model for the accurate computation of the lateral scattering of protons water", Physics Medicine \& Biology, 2016, vol. 61, N102-N117.

[13] J. Kempe and A. Brahme, "Energy-range relation and mean energy variation therapeutic particle beams", Medical Physics, 2008, vol. 35, no.1, 159-170.

[14] W. Ulmer, "Theoretical aspects of energy-range relations, stopping power and energy straggling of protons", Radiation Physics and Chemistry, 2007, vol. 76, 1089-1107.

[15] M. Pavlovič, M. Miglierini, E. Mustafin, W. Ensinger, A. Šagátová and M. Šoka, "Radiation damage studies of soft magnetic metallic glasses irradiated with high-energy heavy ions", Radiation Effects and Defects Solids, 2015, vol. 170, no. 1, 1-6.

[16] T. C. Hufnagel, C. A. Schuh and M. L. Falk, "Deformation of metallic glasses: Recent developments theory, simulations, and experiments", Acta Materialia, 2016, vol. 109, 375-393.

[17] M. Pavlovič, M. Miglierini, E. Mustafin, T. Seidl, M. Šoka and W. Ensinger, "Influence of high energy heavy ions on magnetic susceptibility of soft magnetic metallic glasses", Acta Physica Polonica A, 2014, vol. 126, no.1, 54-55.

[18] M. Miglierini, A. Lančok and M. Pavlovič, "CEMS studies of structural modifications of metallic glasses by ion bombardment", The Physics of Metals and Metallography, 2010, vol. 109, no.5, 469-474.

[19] V. V. Ovchinnikov, F. F. Makhin'ko, N. V. Gushchina, A. V. Stepanov, A. I. Medvedev, Y. N. Starodubtsev, V. A. Kataev, V. S. Tsepelev and V. Y. Belozerov, "Effect of ion irradiation on the nanocrystallization and magnetic properties of soft magnetic Fe72. 5Cu1Nb2Mo1. 5Si14B9 alloy", Physics of Metals and Metallography, 2017, vol. 118, no. 2, 150-157.

[20] S. N. Kane, M. Shah, M. Satalkar, K. Gehlot, P. K. Kulriya, D. K. Avasthi, A. K. Sinha, S. S. Modak, N. L. Ghodke, V. R. Reddy and L. K. Varga, "Modification of structural and magnetic properties of soft magnetic multi-component metallic glass by $80 \mathrm{MeV}$ O-16(6+) ion irradiation", Nuclear Instruments and Methods Physics Research B, 2016, vol. 379, 242-245.

21] Š. Michalík, J. Michalíková, M. Pavlovič, P. Sovák, H. P. Liermann and M. Miglierini, "Structural modifications of swift-ionbombarded metallic glasses studied by high-energy X-ray synchrotron radiation", Acta Materialia, 2014, vol. 80, 309-316.

[22] E. Mustafin, T. Seidl, A. Plotnikov, I. Strak, M. Pavlovi?, M. Miglierini, S. Stan?ek, A. Fertman and A. Lančok, "Ion irradiation studies of construction materials for high-power accelerators", Radiation Effects and Defects Solids, 2009, vol. 164, no.7-8, 460-469.

23] I. Bartošová, J. Veterníková and V. Slugeň, "Study of candidate materials for new reactor systems using positron annihilation spectroscopy and Barkhausen noise", Nuclear Engineering and Design, 2014, vol. 273, 376-380.

24] J. Šimeg, J. Degmová, M. Veterníková, F. Šimko, M. Pekarčíková, M. Petriska, P. Skarba, M. Mikula and Pupala, "Thermal stability study for candidate stainless steels of GEN IV reactors", Applied Surface Science, 2016, vol. 387, 965-970.

25] J. Veterníková, J. Degmová, F. Šimko, M. Pekarčíková, S. Soják and V. Slugeň, "Positron study of steel NF 709 after irradiation and thermal strain", Nuclear Instruments and Methods Physics Research B, 2015, vol. 365, 309-312.

26] S. Soják, J. Šimeg, V. Slugeň, M. Veterníková, M. Petriska and Stacho, "Ion implantations of oxide dispersion strengthened steels", Nuclear Instruments and Methods Physics Research B, 2015, vol. 365, 305-308.

27] J. Š. Veterníková, J. Degmová, V. Sabelová, M. Petriska and V. Slugeň, "Surface study of radiation damaged oxide dispersion strengthened steels", Applied Surface Science, 2014, vol. 312, 199-202.

Received 3 March 2017

Márius Pavlovič (Prof, Dipl Ing, PhD), born in Martin in 1963, Slovakia, graduated from Slovak University of Technology in Bratislava, Faculty of Electrical Engineering and Information Technology in 1986. PhD-degree attained in 1994, from 2001 to 2011 an Assoc Prof, since 2011 full Professor. Main field of research is directed to medical applications of particle accelerators. Awarded by the Award of Science and Technology 2005 of the Ministry of Education, Slovak Republic. Presently a prof. at the Institute of Nuclear and Physical Engineering, FEI STU in Bratislava. Visiting scientist and consulting positions at CERN, GSI Darmstadt, JINR Dubna and Fotec Research and Technology, Ltd. Wiener Neustadt.

Andreas Hammerle holds a Bachelor of Science degree in Radiologic Technologies. He earned his Master of Science degree in MedTech Functional Imaging, Conventional and Ion Radiotherapy in 2016. He was graduated from the University of Applied Sciences in Wiener Neustadt, Austria. Since then, he is working in the field of hearing implant devices at MEDEL Elektromedizinische Gerte GmbH in Austria. 